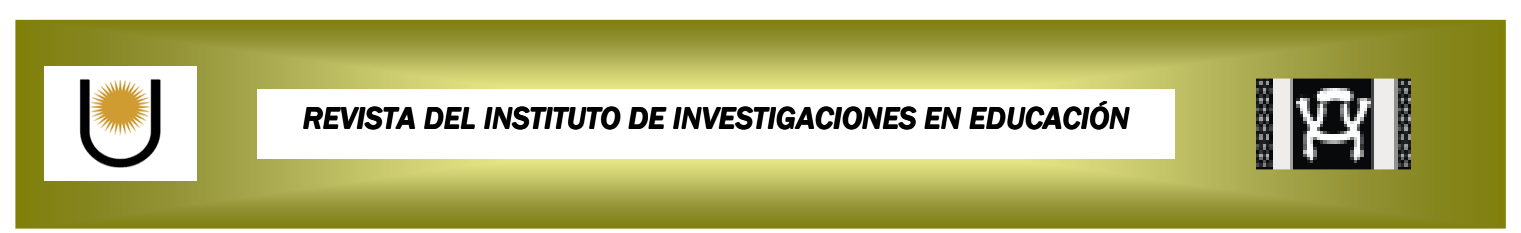

Revista digital. Año 5 - No 5 - Año 2014 - pág. 1 - 49 ISSN $1853-1393$

Resistencia. Chaco. Argentina. 2014

\title{
RAZONAMIENTO PREDICTIVO DE ESTUDIANTES UNIVERSITARIOS EN CONTEXTOS DE FORMACIÓN EN BIOLOGÍA
}

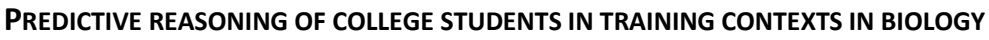

\section{Dr. Aníbal Roque Bar ${ }^{1}$}

Fecha de recepción: 29-05-2014

Fecha de aceptación y versión final: 12-08-2014

Resumen: El presente se propone revelar los modos en que se expresa la inferencia predictiva en estudiantes universitarios de biología.

La muestra está conformada por veinticinco alumnos del último nivel de la carrera. El instrumento de recolección de datos está constituido por dos tareas, la primera de ellas requiere la elaboración de predicciones al modo deductivo clásico, en tanto que la segunda lo hace mediante el uso de análogos.

Los estudiantes muestran mediana eficacia predictiva, y dicha capacidad no difiere sustancialmente si se realiza de manera deductiva o analógica. Se advierte que las relaciones directas o inversas entre los factores incidentes, la influencia del tiempo cronológico en los sucesos, y la complejidad del contenido, afectan la asertividad de la predicción.

Palabras claves: Biología - Predicción - Estudiantes

Summary: This research paper proposes to reveal the ways in which the predictive inference is expressed in college biology students.

The sample consisted of twenty-five students in the final level. The data collection instrument consists of two tasks, the first of which requires making predictions classic deductive approach, whereas the second does by using analogues.

Students show median predictive efficiency, and this capacity is not substantially different if done deductively or analogically. It is noted that the direct or inverse relationships between the contributing factors, the influence of chronological time in the events, and the complexity of content, assertiveness affect the prediction.

Keywords: Biology - Prediction - Students

\footnotetext{
${ }^{1}$ Instituto de Investigaciones en Educación. Facultad de Humanidades. UNNE. Av. Las Heras 727. Resistencia (Chaco). Tel. 03624427470. anibalrbar@hum.unne.edu.ar Profesor Adjunto Regular Cátedra Biología del Aprendizaje. Facultad de Humanidades UNNE.
}

Revista del Instituto de Investigaciones en Educación. Año 5 - Nº5 - Año 2014 - pág. 5 - 15 ISSN $1853-1393$

Resistencia - Chaco - Argentina 


\section{Introducción}

Según Saiz (2002), el razonamiento hipotético "consiste en buscar una explicación, proporcionar una hipótesis, derivar predicciones y probarlas". En este marco la función predictiva es una de las instancias necesarias entre los procesos de producción y contrastación de conocimiento. Se entiende por predicción todo acto enunciativo de anticipación o antelación a los hechos, acto entendido como partir de una hipótesis o supuesto para derivar consecuencias.

La capacidad predictiva de una teoría se mide en términos de su éxito o fracaso en la contrastación empírica, si bien dicho valor se halla condicionado por una serie de factores que relativizan su valoración, como por ejemplo la presencia de "variables ocultas", o la incidencia de "dinámicas complejas" (Bunge, 2001).

Desde la perspectiva de Hempel (2005), la función más importante de las leyes en el campo de las ciencias naturales es la de relacionar hechos con pautas, lo que se encarna en dos clases de enunciados, los predictivos y los explicativos. En la versión hempeliana, la explicación versa sobre un hecho ya acontecido sobre el que debe enunciarse sus condiciones determinantes, lo que se invierte en el caso de la predicción, donde las condiciones iniciales están dadas, y deben afirmarse sus consecuencias.

Dada la semejanza estructural entre explicación y predicción, puede decirse que toda explicación es incompleta, en tanto no pueda funcionar también como predicción. Para Hempel la diferencia entre ambas reside en sus aspectos pragmáticos, pues los requisitos que debe reunir todo acto explicativo son comunes con los exigidos para cualquier situación predictiva. La explicación hempeliana, y por ende la predicción, consta de dos partes: el explanans y el explanandum. La primera constituida por dos clases de afirmaciones, las que formulan las condiciones antecedentes del fenómeno, y las que representan las leyes generales; en tanto que el explanandum se forma por las oraciones que describen el fenómeno. Así, el autor señala cuatro condiciones necesarias para la explicación y la predicción: tres de ellas lógicas, y una empírica. Las condiciones lógicas de adecuación afirman: 1) el explanandum debe ser una consecuencia lógica del explanans; 2) el explanans debe contener leyes generales para la derivación del explanandum; 3) el explanans debe tener contenido observacional. En cuanto a la condición empírica, ésta refiere a la verdad que debe estar contenida en las afirmaciones del explanans.

Desde las epistemologías clásicas la predicción es un acto eminentemente deductivo, pues parte de premisas generales o universales que se aplican a casos particulares para inferir resultados. En esta concepción la verdad de las premisas se conserva necesariamente en la conclusión, cuestión que se sostiene sobre principios lógicos o de vínculos entre proposiciones. Esta perspectiva no pone usualmente en examen el valor veritativo de las premisas, sólo puede asumir que son verdaderas (independientemente de que lo sean), y si opera en estos términos su conclusión también es verdadera. Así, el razonamiento predictivo en su versión lógica soslaya el contenido de los enunciados, deteniéndose exclusivamente en la estructura del argumento.

Cuando el conocimiento en juego está puesto en premisas cuya verdad no se puede afirmar de modo taxativo, la lógica entiende que la conclusión queda indeterminada. Esta imposibilidad de predicción lógica puede subsanarse si se concibe a la proposición de partida como verdadera hasta que nueva evidencia o información colabore con el conocimiento sobre su valor de verdad. Este tipo de inferencia no es un razonamiento estrictamente deductivo sino revocable, lo que implica que opera desde presunciones o premisas de presunta verdad. Para Legris (2002), las inferencias revocables son razonamientos no deductivos donde la conclusión deviene de premisas que constituyen condiciones insuficientes para inferirla, razonamientos habituales en un gran número de situaciones cotidianas entre las que se incluyen la investigación científica o el diseño de artefactos.

Revista del Instituto de Investigaciones en Educación. Año 5 - №5 - Año 2014 - pág. 5 - 15 ISSN $1853-1393$

Resistencia - Chaco - Argentina 
Otra forma de predicción inscrita en el marco de los razonamientos revocables es el que se realiza mediante el uso de analogías. Fernández, González y Moreno (2003) afirman que la analogía consiste en determinar una serie de relaciones de semejanza entre los componentes de la estructura de un análogo y el tópico, a fin de alcanzar la comprensión de este último. Se entiende por análogo al núcleo de la analogía, o sea aquel que comprende lo ya conocido; el tópico refiere a los contenidos que no se conocen; y la trama o relación analógica, al conjunto de las relaciones que posibilitan comparar el análogo con el tópico.

La función de la analogía se comprende al analizar las relaciones entre modelos conceptuales y mentales. Los primeros son representaciones científicas elaboradas con el objeto de facilitar la comprensión y comunicación en la comunidad científica (Moreira, 2002). Dicha comprensión se logra a instancias de construir una representación interna o modelo mental semejante estructuralmente al modelo científico (Johnson-Laird, 1983). En estos términos, los modelos conceptuales están en el dominio de quien enseña o comunica, y el modelo mental en el de quien aprende o recibe la comunicación (González, 2005). Así, ambos modelos son análogos en tanto las relaciones que puedan establecerse entre ambos sean de semejanza estructural. La analogía como tal propicia la comprensión, a la vez que da elementos para esbozar una explicación, y desde allí establecer predicciones. En este sentido, afirma Oliva (2004: 377) que “...las analogías pueden resultar también útiles al elaborar predicciones sobre determinado tipo de fenómenos o también para llevar a cabo una interpretación de los mismos, aunque sólo sea a un nivel figurativo desde una primera aproximación

Gutiérrez (2005) afirma que los modelos mentales de Johnson-Laird coinciden con los formulados por Norman (1983), en dos aspectos, sus objetivos y sus elementos constitutivos. Los primeros son de índole funcional y aluden a las explicaciones y predicciones que se pretenden hacer sobre y desde el sistema físico modelizado. Por su parte, los componentes comprenden dos tipos de representaciones, la primera construida sobre los estados del mundo, y la segunda derivada de ella, definida por un sistema de inferencias que le da condiciones para anticiparse a futuras fases del sistema que se modeliza. Esta última puede sustanciarse de manera mental, por lo que puede poner en relación al sistema modelizado con el sistema físico real. Así, el primer nivel de representaciones implicará una ontología según las creencias e intereses del sujeto, en tanto que el segundo nivel referirá a una epistemología que permitirá establecer tanto los criterios de verdad, como la correspondencia entre la realidad y lo inferido.

Desde la psicología cognitiva se asume que la construcción de un modelo mental integra propiedades, objetos y relaciones que pueden o no tener su correlato con una imagen física. Esto último se da cuando un sujeto imagina a los objetos causando cambios sobre otros, identificando así los aspectos dinámicos de los fenómenos físicos y construyendo representaciones de dichas acciones (Schwartz, 1999). Del mismo modo, otros modelos mentales que producen predicciones exitosas pueden hacer uso de datos conceptuales y perceptivos sin involucrar conocimiento sobre relaciones causales, (Otero, Greca y Lang da Silveira, 2003)

Al decir de Greca y Moreira (1998, pág. 108), "Ni los modelos mentales resultan copias perfectas de los modelos conceptuales que los científicos y profesores generan, ni ese proceso de modelización resulta tan evidente para nuestros alumnos".

Los estudios empíricos sobre predicciones muestran que los sujetos operan con información contextual devenida del conocimiento personal, o bien de la información disponible al momento de la inferencia. Así, Kahneman y Tversky $(1972,1973)$ diseñan tareas donde los sujetos anticipan consecuencias partiendo de dos tipos de información, una distribucional que comunica cómo se distribuye la población en relación con cierta variable en estudio, y otra diagnóstica que aporta

Revista del Instituto de Investigaciones en Educación. Año 5 - №5 - Año 2014 - pág. 5 - 15 ISSN $1853-1393$

Resistencia - Chaco - Argentina 
datos vinculados con el comportamiento de esa misma variable en casos de esa población. Los autores hallan que los sujetos prestan menor atención a las fuentes de información distribucional que a la información diagnóstica, resultado que se revierte cuando la predicción es coincidente con las expectativas previas (Nisbett y Borgida, 1975; Nelson, Biernat y Manis, 1990). Los estudios citados, a diferencia de investigaciones realizadas con tareas descontextualizadas (Piaget e Inhelder, 1951; Edwards, 1968; Cohen, 1979), se estructuraron conforme con contenido temático cotidiano, cuestión que induce a sesgos a la hora de valorar la información y poner en juego la predicción. Tales sesgos se relacionan con teorías implícitas o esquemas que influyen a la hora de interpretar la información disponible (Martin y Little, 1990; Martin, Eisenbud y Rose, 1995).

Castañeda y Rodrigo (2001), estudian el comportamiento predictivo de sujetos en diferentes edades, concluyendo que a menor edad los juicios se modifican en función de la información distribucional, aunque con prioridad en la idiosincrática o autogenerada, y secundariamente en la diagnóstica.

Los estudios precedentes dan cuenta de las funciones que asume la predicción, tanto en el contexto científico como en el cotidiano y, en este último, hacen explícito los sesgos que induce el contenido a la hora de enunciar futuras ocurrencias.

Ante tales resultados vale interrogarse si dichos sesgos siguen siendo igualmente determinantes en contextos académicos, donde el contenido está fuertemente impregnado por conceptos o nociones del ámbito científico. Suele atribuirse a dichos ámbitos un mayor y mejor desarrollo de las funciones lógicas, además de una rigurosidad expresa que los distancia taxativamente de los procesos y contenidos del sentido común. Uno de tales contextos es el de la formación disciplinar universitaria, en el cual las teorías y leyes científicas ocupan un lugar primordial, sobre todo cuando dicho marco se adscribe a un campo normativo y fuertemente monoparadigmático como lo es el de las ciencias naturales, y en ellas la física, la química o la biología, entre otras (Biglan, 1973; Echeverría, 1998). En este sentido, algunas investigaciones muestran que los estudiantes de biología muestran gran capacidad en tareas lógicas cuando éstas se organizan sobre contenido disciplinar acotado y sin márgenes para su libre interpretación, (Bar y Ortíz, 2011; Corral y Bar, 2012).

En razón de lo expuesto, es objetivo del presente revelar los modos que asume la predicción en ámbitos de la formación disciplinar inicial, particularmente en estudiantes de las carreras que enseñan biología (profesorado y licenciatura) en contexto universitario. Más específicamente, se prevé analizar cómo se desenvuelve la predicción cuando se realiza a partir de premisas con contenido disciplinar, tanto a la manera deductiva clásica, como desde un análogo.

\section{Materiales y Métodos}

La muestra está conformada por veinticinco estudiantes, 14 mujeres y 11 varones de entre 21 y 26 años de edad, cursantes de cuarto o quinto año de la carrera de biología (profesorado o licenciatura) con el ciclo básico aprobado, pertenecientes a una universidad pública, y que prestaron su conformidad para la resolución de las tares propuestas. Este criterio de inclusión se define sobre el supuesto de que dichos sujetos conocen debidamente los contenidos específicos, como asimismo las operaciones que la disciplina inculca durante la formación, y en tanto tales, son referentes o informantes idóneos a tal fin.

El instrumento de recolección de datos está constituido por dos tareas, la primera de ellas se inicia con la presentación de un texto ad-hoc que describe el proceso de transpiración foliar, y plantea una hipótesis explicativa que indica los modos en que se relaciona la evaporación en los vegetales con las variables climáticas y demás factores contextuales. El mismo se hallaba redactado de la siguiente manera:

Revista del Instituto de Investigaciones en Educación. Año 5 - №5 - Año 2014 - pág. 5 - 15 ISSN $1853-1393$

Resistencia - Chaco - Argentina 
"La transpiración es un proceso mediante el cual la planta evapora el agua acumulada en el parénquima de las hojas a través de los estomas foliares. Los estomas se abren en la cutícula, capa impermeable que la rodea completamente.

La transpiración es básicamente un proceso por el cual se regula la cantidad de agua en el marco de cierto rango, variable según el tipo de vegetal. El mismo es dependiente no sólo de la capacidad que tiene la planta para absorber agua, sino también del nivel de humedad que retiene el suelo, ya que aquellos más porosos o permeables disponen de agua por menos tiempo.

Las células estomáticas operan mediante mecanismos vinculados con la presión del agua al interior del parénquima foliar. A mayor presión los estomas se abren, permitiendo la evaporación. Cuando ésta disminuye, se cierran, evitándola. La cutícula por su parte, también colabora en el proceso, ya que al incrementar su grosor, incrementa también su nivel de impermeabilidad. La temperatura y la humedad atmosférica son también factores que coadyuvan de modo inverso: el calor favorece la evaporación, en tanto que la humedad la dificulta".

La temática tratada en el texto precedente corresponde al ámbito de la fisiología vegetal, contenido que los alumnos conocen desde los primeros años de la carrera.

De dicho texto se extraen dieciséis situaciones presentadas en ocho pares, que combinan información sobre diferentes espacios y tiempos, donde cada par alude a un mismo espacio en dos tiempos diferentes, o bien a dos espacios en un mismo momento. El comportamiento de cada espacio o tiempo que se compara es necesariamente opuesto a lo que se espera del otro componente del mismo par.

Los datos espaciotemporales refieren a siete formaciones fitogeográficas (desierto, selva subtropical, selva amazónica, tundra, estepa siberiana, oasis sahariano, y meseta patagónica), dos fases del día (mañana y noche), cuatro tipos de suelo (arcilloso, pedregoso, anegado y seco), y cuatro localizaciones en el espacio (próxima y alejada de un cuerpo de agua, en la costa marítima y lejos de ella). Debe hacerse explícito que si bien la explicación precedente a la solicitud de predicción da cuenta debida del mecanismo operante, no describe en todos los casos las situaciones particulares para cada uno de los espacios referidos, lo que requiere del conocimiento previo de los mismos. Dicho de otro modo, se hace necesario disponer de ciertos saberes disciplinares para la elaboración de la respuesta a cada ítem, particularmente de aquellos en vínculo con las formaciones fitogeográficas.

Ante cada situación descrita los estudiantes deben establecer predicciones sobre el comportamiento de los estomas foliares y sobre el nivel de evaporación o transpiración de la planta en esos contextos. La pregunta que orienta la tarea se halla formulada de la siguiente manera:

"Dadas las siguientes situaciones comparadas, imagina qué cabría esperar ocurra en plantas de diferentes ambientes, tanto en el nivel de apertura de los estomas como en el nivel de evaporación o transpiración de la vegetación:

a) Un desierto en la noche y en la mañana.

b) La selva subtropical en la mañana y en la noche.

c) Una zona próxima a un cuerpo de agua en la selva amazónica, y otra alejada de ríos o lagunas en el mismo ambiente.

c) La tundra en el día y en la noche.

d) La estepa siberiana y el oasis sahariano.

e) La meseta patagónica, en la costa marítima y alejada de ella.

f) Una planta en suelo pedregoso, y otra de la misma especie en suelo arcilloso.

g) Una planta en suelo anegado, y otra de la misma especie en suelo seco".

Revista del Instituto de Investigaciones en Educación. Año 5 - ํ5 - Año 2014 - pág. 5 - 15 ISSN $1853-1393$

Resistencia - Chaco - Argentina 
Se consideraron correctas sólo aquellas respuestas donde ambas predicciones del mismo par fueron coincidentes con lo que se esperaba en términos lógicos y empíricos, considerándose incorrectas a aquellas donde ninguna o sólo una, fueran coincidentes con lo previsto.

La segunda tarea se formula con base en un texto que describe el funcionamiento de un dispositivo mecánico, instrumento análogo a un vegetal, que sirve de modelo para explicar el proceso de transpiración foliar en las plantas. En este caso la regla que explica el proceso en el dispositivo debe ser generada por los estudiantes, tomando como análoga la explicación dada en la primera tarea. Dicho texto se halla formulado de la siguiente manera:

"La transpiración de las plantas suele modelizarse mediante un dispositivo físico que simula los fenómenos de absorción, transporte y evaporación implicados en dicho proceso. El mismo consiste en un tubo capilar cuya abertura inferior se introduce en el suelo, en tanto que la superior se cubre con papel celofán poroso"

Al finalizar la transcripción del texto precedente, se presenta la siguiente cuestión:

“¿Puedes identificar en dicho dispositivo los elementos análogos a los de las plantas? Si puedes hacerlo, elabora dos listados, uno a la izquierda de la página (con los elementos de la planta), y otro a la derecha (con los elementos del dispositivo físico), mostrando las correspondencias entre ellos".

Finalizada la primera parte de la segunda tarea, se presenta la siguiente consigna:

"Indica lo que ocurriría en el dispositivo físico (en términos del nivel de evaporación o transpiración), en las siguientes situaciones, suponiendo que el resto de las condiciones no varie:

a) introducido en suelo arenoso y en suelo arcilloso;

b) constituido por celofán mucho y poco poroso;

c) sometido a excesiva y a escasa humedad atmosférica;

d) sometido a elevada y a baja temperatura".

Del mismo modo que en la primera tarea, sólo se consideraron correctas aquellas respuestas donde ambas predicciones del mismo par fueron coincidentes con lo previsto por la lógica y la disciplina.

\section{Resultados}

Para la primera tarea, $54 \%$ de los estudiantes en promedio responde coincidentemente con lo esperado; no sólo en términos lógicos, sino también desde el conocimiento disciplinar.

Cuando la predicción se efectúa a partir de la comparación de los mismos espacios geográficos en dos secuencias temporales (tres situaciones), la proporción promedio de respuestas correctas se ubica por debajo de la media (35\%), según se muestra en el siguiente cuadro:

\section{Cuadro $\mathbf{N}^{\circ}$ 1. Número de estudiantes agrupados según calidad de las predicciones sobre el comportamiento foliar en tres espacios geográficos y dos tiempos diferentes. n: 25}

\begin{tabular}{|c|c|c|c|c|c|c|}
\hline \multirow[t]{2}{*}{ Calidad predictiva } & \multicolumn{2}{|c|}{$\begin{array}{l}\text { Desierto durante el día y } \\
\text { durante la noche }\end{array}$} & \multicolumn{2}{|c|}{$\begin{array}{l}\text { Selva durante el día y } \\
\text { durante la noche }\end{array}$} & \multicolumn{2}{|c|}{$\begin{array}{l}\text { Tundra durante el día y } \\
\text { durante la noche }\end{array}$} \\
\hline & $\mathrm{n}$ & $\%$ & $\mathrm{n}$ & $\%$ & $\mathrm{n}$ & $\%$ \\
\hline Correcta & 15 & 60 & 6 & 24 & 5 & 20 \\
\hline Incorrecta & 10 & 40 & 19 & 76 & 20 & 80 \\
\hline Total & 25 & 100 & 25 & 100 & 25 & 100 \\
\hline
\end{tabular}

Revista del Instituto de Investigaciones en Educación. Año 5 - Nº - Año 2014 - pág. 5 - 15 ISSN $1853-1393$

Resistencia - Chaco - Argentina 
Los aciertos en la predicción se sitúan por encima de la media (66\% en promedio) cuando se realizan a partir del análisis de casos en los que se comparan dos espacios en un mismo momento (cinco situaciones). Debe destacarse que tres de ellas refieren a espacios complejos (formaciones fitogeográficas) identificados por una gran número de factores incidentes; en tanto que dos situaciones aluden a espacios restringidos (tipos de suelo), distinguidos por un número reducido de propiedades. Los resultados para cada caso se expresan en el cuadro que se presenta a continuación:

Cuadro $\mathrm{N}^{\circ}$ 2. Cantidad de estudiantes agrupados según predicciones sobre el comportamiento foliar en cinco espacios y un mismo tiempo. n: 25

\begin{tabular}{|c|c|c|c|c|c|c|c|c|c|c|}
\hline \multirow[t]{2}{*}{$\begin{array}{l}\text { Calidad } \\
\text { predictiva }\end{array}$} & \multirow{2}{*}{\multicolumn{2}{|c|}{$\begin{array}{l}\text { Selva tropical. } \\
\text { Próximo y } \\
\text { lejano al agua } \\
n \quad \%\end{array}$}} & \multicolumn{2}{|c|}{$\begin{array}{l}\text { Estepa } \\
\text { siberiana y } \\
\text { oasis sahariano }\end{array}$} & \multicolumn{2}{|c|}{$\begin{array}{l}\text { Meseta } \\
\text { patagónica. } \\
\text { Próximo y } \\
\text { lejano al mar }\end{array}$} & \multicolumn{2}{|c|}{$\begin{array}{l}\text { Suelo } \\
\text { pedregoso y } \\
\text { arcilloso }\end{array}$} & \multicolumn{2}{|c|}{$\begin{array}{l}\text { Suelo anegado } \\
\text { y seco }\end{array}$} \\
\hline & & & $\mathrm{n}$ & $\%$ & $\mathrm{n}$ & $\%$ & & $\%$ & $n$ & $\%$ \\
\hline Correcta & 13 & 52 & 15 & 60 & 14 & 56 & 20 & 80 & 20 & 80 \\
\hline Incorrecta & 12 & 48 & 10 & 40 & 11 & 44 & 5 & 20 & 5 & 20 \\
\hline Total & 25 & 100 & 25 & 100 & 25 & 100 & 25 & 100 & 25 & 100 \\
\hline
\end{tabular}

Lo reseñado parece mostrar que el acto predictivo resulta más dificultoso cuando emerge de comparaciones donde el tiempo opera como factor de análisis. Del mismo modo, cuando la anticipación se realiza desde contenidos que refieren a espacios restringidos, tal los tipos de suelo (80\%), resultan claramente más sencillas que cuando se efectúan desde aquellos más extensos y complejos como lo son la selva, la estepa y la meseta (56\% en promedio). En este caso, aplicado el test de $X^{2}$ se obtiene el valor $X^{2}(24)=16,66 p<0,03$, el que da muestras de la alta significación de las diferencias entre valores teóricos y empíricos.

En lo que hace a la segunda tarea, $60 \%$ de los estudiantes logra establecer analogías correctas entre los componentes de la planta y el dispositivo mecánico, o sea que la raíz, el tallo y las hojas del vegetal son análogos a la abertura inferior del tubo capilar, a su porción central, y a su extremo superior cubierto por papel celofán, respectivamente. El resto de los alumnos vincula sólo los dos últimos pares de análogos (32\%), o bien el segundo exclusivamente (8\%).

Identificados los componentes análogos de la planta con el dispositivo, 64\% de los estudiantes (en promedio) logra hacer predicciones correctas, según se desprende del análisis del siguiente cuadro:

Cuadro $\mathrm{N}^{\circ}$ 3. Número de estudiantes agrupados según calidad predictiva sobre el comportamiento del dispositivo mecánico en cuatro situaciones comparadas. n: 25

\begin{tabular}{|c|c|c|c|c|c|c|c|c|}
\hline Calidad predictiva & \multicolumn{2}{|c|}{$\begin{array}{l}\text { Suelo arenoso y } \\
\text { arcilloso }\end{array}$} & \multicolumn{2}{|c|}{$\begin{array}{l}\text { Celofán muy } \\
\text { poroso y poco } \\
\text { poroso }\end{array}$} & \multicolumn{2}{|c|}{$\begin{array}{l}\text { Humedad } \\
\text { atmosférica escasa } \\
\text { y excesiva } \\
\text { n } \%\end{array}$} & \multicolumn{2}{|c|}{$\begin{array}{l}\text { Temperatura } \\
\text { elevada y baja }\end{array}$} \\
\hline Correcta & 15 & 60 & 23 & 92 & 3 & 12 & 23 & 92 \\
\hline Incorrecta & 9 & 36 & 1 & 4 & 21 & 84 & 1 & 4 \\
\hline No responde & 1 & 4 & 1 & 4 & 1 & 4 & 1 & 4 \\
\hline Total & 25 & 100 & 25 & 100 & 25 & 100 & 25 & 100 \\
\hline
\end{tabular}

Revista del Instituto de Investigaciones en Educación. Año 5 - ํo5 - Año 2014 - pág. 5 - 15 ISSN $1853-1393$

Resistencia - Chaco - Argentina 
Según se deriva de la lectura del cuadro precedente, la humedad atmosférica como variable incidente en los procesos de transpiración, opera como factor cuyo análisis ofrece alguna dificultad a la hora de hacer predicciones correctas.

\section{Discusión y Conclusiones}

En el campo de las ciencias naturales, dada su matriz fuertemente monoparadigmática (Biglan, 1973), la predicción asume un rol preponderante, toda vez que habilita a anticipar resultados con altos niveles de certeza, cuestión no menor desde la perspectiva de una ciencia positiva cual es la biología, ni tampoco desde la mirada del sujeto que la aprende en el ámbito de la formación inicial, los estudiantes de dicha carrera.

La primera tarea solicitada a los alumnos se estructuró de acuerdo con la forma deductiva clásica (Hempel, 2005), es decir la presentación del explanans expresado en leyes (las de la absorción, el transporte y la transpiración foliar en vegetales) y en condiciones antecedentes (un desierto en la noche y en la mañana...), a los cuales se debía incorporar el explanandum como respuesta. Cada predicción correcta de los alumnos cumplió con las cuatro condiciones de adecuación exigidas por Hempel; es lo que ocurrió en aquellas situaciones en las cuales la información puesta en el explanans era suficiente para derivar el explanandum, tal el caso de las inferencias que tomaron como punto de partida los tipos de suelo, donde los niveles de acierto alcanzaron $80 \%$.

En virtud de lo hallado en el presente y contrario a lo encontrado en otras investigaciones (Bar y Ortíz, 2011; Corral y Bar, 2012), las predicciones que realizan los alumnos de biología no se presentan en el mejor nivel cuando éstas se efectivizan siguiendo el modelo deductivo clásico (54\% de predicciones correctas). Dicho resultado se debilita aún más cuando la dimensión temporal opera como factor incidente en el fenómeno que se analiza, y se fortalece cuando éste está ausente. Es posible que dicho comportamiento esté dado por improntas de la formación disciplinar que favorecerían actividades áulicas con análisis transeccionales por sobre los longitudinales. Si esto es así, los procesos formativos no daría elementos suficientes para construir una representación adecuada del "transcurso del tiempo" como factor de incidencia sobre los estados del mundo, lo que afectaría las inferencias a futuro sobre dichos estados (Gutiérrez, 2005).

Cuando los alumnos realizan predicciones en relación con el contexto del desierto, éstas resultan menos sesgadas que cuando se efectúan sobre ámbitos como la tundra o la selva. A que se debe dicha diferencia? Las variables ambientales cuyo comportamiento se describe en el texto para el análisis son sólo dos, la temperatura y la humedad atmosférica. Pareciera que la evaluación del comportamiento de esta última por parte de los estudiantes ofrece alguna dificultad adicional no presente al momento de predecir la acción térmica. En el desierto, dada la ausencia de humedad, la temperatura es el único factor ambiental (según el texto ofrecido para la tarea) que puede alterar la apertura de los estomas y la consiguiente evaporación, a diferencia de lo que ocurre en la tundra y en la selva, donde ambas variables interactúan entre sí y con la planta. Esta última situación no es sólo más compleja, sino que adiciona un posible problema de comprensión acerca de cómo verdaderamente funciona la humedad al momento de la transpiración.

Las diferencias en la acción de ambos factores son esencialmente diferentes. El calor genera que el agua en estado fluido se transforme en vapor. El exceso de humedad por su parte, en tanto gas ya presente en la atmósfera con altos niveles de presión, hace que el agua en estado líquido en el parénquima foliar no pueda alcanzar el estado gaseoso. Así, la temperatura covaría con la evaporación, asumiendo una relación matemática de tipo directa con pendiente positiva; en tanto que la misma en vínculo con la humedad atmosférica, toma la forma de pendiente negativa. La

Revista del Instituto de Investigaciones en Educación. Año 5 - №5 - Año 2014 - pág. 5 - 15 ISSN $1853-1393$

Resistencia - Chaco - Argentina 
correlación negativa supone una suerte de inhibición de una variable respecto de la otra, o bien la idea de que la variable dependiente no llega a comportarse según lo previsible en presencia de la variable independiente. Vale mencionar que de todos los factores nominados, tanto ambientales como vegetales, la humedad atmosférica es el único que guarda relaciones negativas con las demás variables cuantitativas en concurso. Así, es posible argumentar que la dirección de la relación que se establece entre los factores incidentes en el fenómeno, es un elemento condicionante a la hora de establecer predicciones. Pareciera que en este caso el primer nivel de representaciones construido por los estudiantes en función de la interacción entre factores, derivó en una ontología que puso obstáculos para la construcción de una epistemología orientada al logro de buenas predicciones (Johnson-Laird, 1983).

Además de la cuestión temporal o de la clase de vínculo entre variables, los contenidos que ofician de premisas inciden de algún modo en la calidad de las predicciones. Así, cuando lo que se compara son espacios geográficos que se diferencian por múltiples variables (estepa, meseta, selva), se exige al estudiante poner en juego conocimientos y relaciones más complejas que cuando la comparación se realiza entre meras localizaciones al interior de un mismo espacio (cerca o lejos de un cuerpo de agua, próximo o lejano al mar), y por lo tanto se incrementan los sesgos de predicción.

A diferencia de un espacio restringido, las formaciones fitogeográficas constituyen tipologías, y como tales suponen la reducción de un sinnúmero de valores de variable a un tipo o clase ideal que no siempre se evidencia en la realidad. Así, aunque el comportamiento de un tipo corresponda a una ley (como parte del explanans), no todo ello es previsible, dado no sólo la idealidad del tipo, sino la contingencia que supone el concurso de un gran número de variables. Es posible que esta particularidad dificulte las predicciones que se realizan sobre estos espacios, puesto que su mera referencia no alcanzaría para definir con claridad los componentes del explanans, lo cual dificultaría la predicción deductiva, y por ende determinística, y lo acercaría más a aquellas en el marco de los razonamientos revocables (Legris, 2002), donde el antecedente no constituye condición suficiente para la derivación del consecuente.

En la segunda tarea, cuando se pide establecer correlaciones entre dos entidades semejantes, se halla explícito que el análogo está constituido por la planta, y el tópico por el dispositivo mecánico. Lo que los estudiantes deben hacer en ese caso es construir la trama de relaciones que conectan los componentes del análogo con los del tópico (Fernández, González y Moreno, 2003). Lo que el texto para el análisis comunica es el modelo conceptual de transpiración foliar aceptado por la disciplina, hallándose en la respuesta de los estudiantes elementos que dan cuenta de sus modelos mentales en relación con el fenómeno que se explica (Johnson-Laird, 1983; González, 2005). En este contexto, la mayoría de ellos logra establecer buenas correspondencias, del mismo modo que cuando deben realizar predicciones tomando como base el modelo análogo. Esta tarea, a diferencia de la primera, no hace explícita la regla componente del explanans, sólo da sus condiciones iniciales, de modo tal que ésta ha debido ser elaborada por los alumnos tomando la explicación de la primera tarea como análoga.

De modo similar a lo ya señalado para las predicciones con estructura deductiva, se advierte que cuando la anticipación se realiza desde premisas con contenidos relativos a "humedad atmosférica", los sesgos se incrementan notablemente. Como ya se explicó para los casos deductivos, no es el contenido en sí mismo el que afecta la inferencia, sino que se trata de dificultades en la comprensión de las relaciones negativas entre variables (a mayor humedad atmosférica, menor evaporación, y viceversa). Pareciera que en estos casos la formación no contribuye al análisis de fenómenos donde las relaciones entre variables, aunque directas, se expresen con pendiente negativa, y con ello se orienta en la definición de una epistemología que no colabora en la generación de predicciones eficientes (Johnson-Laird, 1983).

Revista del Instituto de Investigaciones en Educación. Año 5 - N5 - Año 2014 - pág. 5 - 15 ISSN $1853-1393$

Resistencia - Chaco - Argentina 
La predicción mediante el análogo ha requerido que los estudiantes infieran analogías funcionales no solicitadas previamente, ya que la primera parte de la tarea sólo requería poner en juego correspondencias estructurales. Al respecto vale mencionar que, si bien parte de los alumnos no logró analogar todos los componentes de la planta y del dispositivo ( $40 \%$ de la muestra), esto no ha sido un obstáculo para la predicción solicitada a posteriori, dado que esos mismos estudiantes sí han podido hacer anticipaciones correctas. Lo señalado parece mostrar que la posibilidad de relacionar estructuras análogas no es condición necesaria a la hora de definir correspondencias funcionales, situación que podría explicarse a partir de la concepción de analogía acuñada por la biología. En esta disciplina, lo análogo no está puesto en la semejanza estructural sino en la función, ya que estructuras de diferente naturaleza pueden ejercer acciones en pos de resolver los mismos problemas (Curtis et al, 2007).

A modo de síntesis, puede decirse que los estudiantes de la carrera de biología no han logrado en todos los casos la construcción de modelos mentales en concordancia con los correspondientes conceptuales, y que dicha modelización no ha resultado en los alumnos todo lo elocuente que se esperaba (Greca y Moreira, 1998).

Se advierte además que el tiempo como variable de análisis, los tipos de vínculos entre factores incidentes en el fenómeno, y la complejidad del contenido de las premisas, afectan la asertividad de la predicción. De los elementos señalados se entiende que el primero y el segundo pueden vincularse con sesgos formativos. En lo que hace a la influencia del contenido, pareciera relacionarse más con dificultades en la comprensión de los fenómenos propios del campo disciplinar, que con condicionamientos implícitos.

\section{Bibliografía}

Bar A. R. y Ortíz M. C. (2011). Las habilidades de razonamiento causal de estudiantes universitarios, aplicadas en la interpretación de textos disciplinares. En Cuadernos de Educación, Año 9, núm. 9, $209-217$.

Bunge M. (2001). La investigación científica: Su estrategia y su filosofía. Bs. As: Siglo XXI.

Castañeda J. y Rodrigo M. J. (2001). La influencia del contenido en el razonamiento predictivo: un estudio evolutivo con estereotipos de género. En Anuario de Psicología, Año 32, núm.1, 31- 49.

Cohen, L. J. (1979). On the psychology of prediction. Whose is the fallacy?, En Cognition, Año 7, 385-407.

Corral N. J. y Bar A. R. (2012). Construcción de racionalidades disciplinares,. En Cinta de Moebio. Rev. Elec. de Epistemología de Ciencias Sociales de la Universidad de Chile. Núm. 45, 215 - 234.

Curtis H., Barnes S., Schnek A. y Massarini A. (2007). Biología. Bs. As: Panamericana.

Edward W. (1968). Conservationism in human information processing, en B. Kleinmuntz (Comp), Formal Representation of Human Judgment. New York, John Wiley and Sons, Inc.

Fernández J. González B. y Moreno T. (2003). Las analogías como modelo y como recurso en la enseñanza de las ciencias. En Alambique, Año 35, 82-89.

González B. (2005). El modelo analógico como recurso didáctico en ciencias experimentales. En Revista Iberoamericana de Educación, Año 37, núm. 2, 1-15.

Hempel C. (2005). La explicación científica: Estudios sobre la filosofía de la ciencia. Barcelona: Paidós.

Revista del Instituto de Investigaciones en Educación. Año 5 - №5 - Año 2014 - pág. 5 - 15 ISSN $1853-1393$

Resistencia - Chaco - Argentina 
Johnson-Laird, P. N. (1983). Mental models. Cambridge, M.A: Harvard University Press.

Kahneman, D. y Tversky, A. (1972). Subjective probability: a judgment of representativeness. En Cognitive Psychology, Año 3, 430-454.

Kahneman, D. y Tversky, A. (1973). On the psychology of prediction. En Psychological Review, Año 80, 237-251.

Legris J. (2002). Razonamiento revocable y lógicas no monótonas: un análisis conceptual. En Cuadernos del CIMBAGE, 005, 109-131.

Martin, C.L. y Little, J.K. (1990). The relation of gender understanding to children's sex-typed preferences and gender stereotypes. En Child Development, Año 61, 1427-1439.

Martin, C.L., Eisenbud, L. y Rose, H. (1995). Children's gender-based reasoning about toys. En Child Development, Año 66, 1453-1471.

Moreira, M. A. (2002). Modelos mentales y modelos conceptuales en la enseñanza-aprendizaje de la Física y en la investigación en este campo. En XX Encuentros de Didáctica de las Ciencias Experimentales: 31-47.

Nelson, T. E., Biernat, M. R. y Manis, M. (1990). Everyday Base Rates (Sex Stereotypes): Potent and Resilient. En Journal of Personality and Social Psychology, Año 59, núm. 4, 664-675.

Nisbett, R.E. y Borgida, E. (1975). Attribution and the psychology of prediction. En Journal of Personality and Social Psychology, Año 32, 932-943.

Norman, D.A. (1983). Some observations on mental models, en D. Getner y A.L. Stevens (Ed.), Mental Models. Hilsdalle, NJ, Lawrence Erbaum Associates.

Oliva J. M. (2004). El pensamiento analógico desde la investigación educativa y desde la perspectiva del profesor de ciencias. En Rev. Electrónica de Ens. de las Ciencias, Año 3, núm 3, 363384.

Piaget, J. y Inhelder, B. (1951). La genèse de l'idée de hasard chez l'enfant. Paris: Presses Universitaries de France.

Saiz C. (2002). Pensamiento crítico. Conceptos básicos y actividades prácticas. Madrid: Psicología Pirámide.

Schwarts, D. (1999). Physical Imagery: Kinematic versus Dinamic Models. En Cognitive Psychology, Año 38, 433- 464. 